\title{
OPTIMIZATION OF PLATE RECOGNITION SENSOR LOCATIONS: A CASE STUDY IN TURKEY
}

\author{
Buğra GÖR1,2, Gülșah KARAKAYA2* \\ ${ }^{1}$ McKinsey \& Company, Mustafa Kemal Mah., A blok, No:50, Tepe Prime Çankaya/Ankara \\ ORCID No: https://orcid.org/0000-0002-4545-1150 \\ ${ }^{2}$ Orta Doğu Teknik Üniversitesi, İktisadi ve İdari Bilimler Fakültesi, İşletme Bölümü, Ankara \\ ORCID No: https://orcid.org/0000-0001-9061-103X
}

\begin{tabular}{ll}
\hline Keywords & Abstract \\
\hline Centralized decision- & With the advances in sensor and data transfer technologies, the usage areas of \\
making, network coverage, & Automatic License Plate Recognition (ALPR) systems have been expanded in the public \\
sensor location selection, & and private sectors. In public safety, ALPR systems are used to monitor and control \\
license plate recognition, & $\begin{array}{l}\text { traffic data at both individual and collective levels. To build an efficient sensor } \\
\text { network, the locations of ALPR systems should be determined optimally. This study } \\
\text { provides an approach to determine optimal locations of ALPR systems that maximize } \\
\text { network coverage consisting of two measures: i) vehicle coverage and ii) road } \\
\text { coverage. The former represents the daily average vehicle flow whereas the latter } \\
\text { stands for the number of road-links covered. The relative importance of vehicle and } \\
\text { road coverages are taken into consideration, and optimal solutions under various } \\
\text { scenarios are presented. A close neighbor constraint is introduced to avoid inefficient } \\
\text { distribution of ALPR systems on the network. A case study with numerical examples } \\
\text { designed for two cities in Turkey is provided. The centralized and decentralized } \\
\text { solutions are compared against the current state, and the results show that the } \\
\text { network coverage increases substantially in the centralized case. }\end{array}$ \\
\hline
\end{tabular}

\section{PLAKA TANIMA SENSORÜ YER SEÇIMİ OPTIMIZASYONU: TÜRKIYE ÖRNEĞİ}

\begin{tabular}{|c|c|}
\hline Anahtar Kelimeler & Öz \\
\hline $\begin{array}{l}\text { Merkezi karar verme, ağ } \\
\text { kapsama, sensör yer seçimi, } \\
\text { plaka tanıma, trafik } \\
\text { güvenliği }\end{array}$ & 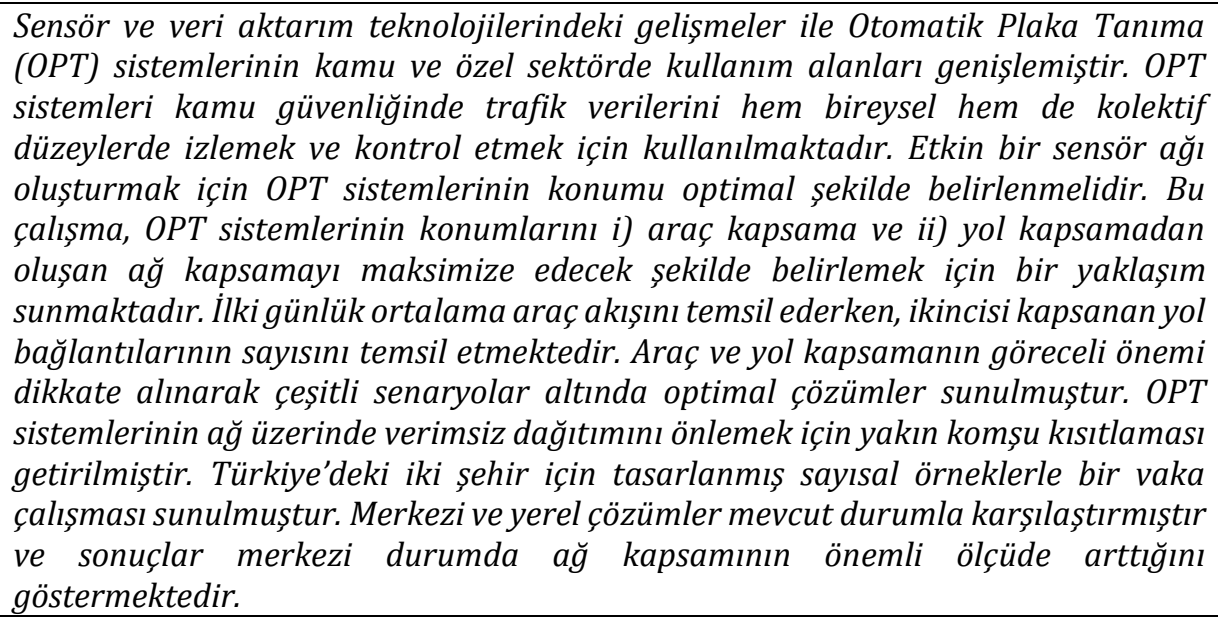 \\
\hline Araştırma Makalesi & Research Article \\
\hline Başvuru Tarihi & Submission Date \\
\hline Kabul Tarihi & Accepted Date \\
\hline
\end{tabular}

*Sorumlu yazar; e-posta: kgulsah@metu.edu.tr 


\section{Introduction}

A couple of years after the Wards Auto's report (2011) on world vehicle population topping 1 billion units in 2010, it surpassed 1.2 billion units in 2014, and it is forecasted that 2 billion vehicles will be on roads by 2035 (Voelcker, 2014). Growing number of vehicles necessitates efficient intelligent transportation system (ITS) solutions to plan and manage traffic. With the advancements in computer technology, traffic surveillance has become one of the main ITS application areas. This, in turn, makes it necessary to collect real-time traffic data via sensor networks for managing and analyzing transportation systems. Various sensor technologies such as surveillance cameras, Radio-Frequency Identification (RFID) readers, speed detecting sensors, image recognition sensors, and loop detectors are used to collect real-time traffic data. Automatic License Plate Recognition (ALPR) systems gain popularity in today's data-driven traffic management as they carry more information than traditional vehicle counting sensors and provide better estimations (Castillo, Menendez and Jimenez, 2008).

ALPR can be defined as a process of capturing images/videos of license plates and transforming the collected visual data to alphanumeric plate numbers through certain algorithms. ALPR technology was developed in the UK in 1976 by the British Police Scientific Development Branch. At that time, accuracy was low due to limited computational capacity and lack of digital cameras. Technological developments in the related areas led to improvements in the hardware capability while expanding the usage areas of these systems for both the public and private sectors. Practices include but not limited to parking lot plate reading, gas station vehicle recognition, toll collection systems, customs control, traffic control, and public safety applications. Terrorist attacks in London in 1993 resulted in the "ring of steel", a surveillance and security cordon supported by closed-circuit television and ALPR cameras that were capable of processing the information and providing feedback to the operator within four seconds (Coaffee, 2004). Law Enforcement Management and Administrative Statistics (LEMAS) surveys in 2007 and 2013 provide insights into the usage rate of ALPR systems in the United States. The rate of law enforcement agencies that use ALPR systems increased from 19\% to $34 \%$ in the period of $2007-2013$, indicating that
ALPR systems were gaining popularity in public safety applications.

In public safety, ALPR systems are typically used to detect and track vehicles associated with certain crimes, such as unregistered vehicles and unpaid traffic tickets. To maximize detection coverage, highpopulated roads are monitored. On the other hand, users of vehicles/plates linked with major crimes (e.g. auto theft, human/drug trafficking, and terrorism) prefer less crowded byroads. This can be associated with crime displacement that is defined as the relocation of a crime as a result of crimeprevention efforts. In order to improve major crime detection rates, the turning points in the road network should be covered; thus, crossroads and junctions should be monitored more closely. In this study, an approach developed to address the efficiency of ALPR networks in terms of network coverage that consists of two measures: vehicle coverage (high-volume) and road coverage (junctions).

In practice, a nation-wide ALPR system network consists of hundreds of cameras and control points distributed to a large road network, and the decision on the quantities and exact locations of those systems is made by local authorities (decentralized) or central authority (centralized). Local authorities are responsible for their own area of jurisdiction, whereas central authority considers nation-wide road network as a whole.

In this study, answers to two questions are sought: 1) What should be the optimal locations of ALPR sensors on a road network to maximize the network coverage? and 2) What is the impact of adopting different decision-making settings (centralized or decentralized) on network coverage for a large road network?

To answer these questions, a bi-objective integer linear model is developed. Weighted sum method is adopted for the objective function in order to find the optimal locations with vehicle coverage and road coverage parameters under different weight settings. The study provides a numerical demonstration of the effects of the two decisionmaking settings, decentralized (DEC) and centralized (CEN), on the network coverage. Models are solved for a case study including two cities in Turkey. The network coverage of the real-life current status is compared to the corresponding DEC and CEN solutions. The differences between improving the existing sub-optimal network and building on an empty network are also discussed. 
The rest of the paper is organized as follows: Section 2 provides the related literature. In Section 3, the approach is developed. A case study with numerical examples on a two-city network is introduced, and experiments are given in Section 4. Results and discussions are presented in Section 5. Finally, in Section 6 , the concluding remarks and future study directions are provided.

\section{Literature Review}

The problem of determining the optimal location of ALPR systems on a road network falls within the general framework of coverage problems under location theory. In the literature, coverage problems are divided into two major categories: Maximum Coverage Location Problem (MCLP) and Set Covering Location Problem (SCLP). In MCLP, the goal is to maximize the satisfied demand with a limited number of facilities available. The objective of SCLP, on the other hand, is to find the minimum number of facilities while meeting the whole demand, i.e., covering the set. It is known that both MCLP and SCLP are NP-hard problems, and various heuristics are developed to solve these problems: Jia, Ordonez and Dessouky (2007) develop a genetic algorithm, a locate-allocate heuristic and a Lagrangian relaxation solution to solve their large scale maximal covering problem with multiple facility quantity-of-coverage and quality-of-coverage requirements. Diaz, Luna, Camacho-Vallejo and Casas-Ramírez (2017) use GRASP (Greedy randomized adaptive search procedure) and hybrid-GRASP-Tabu heuristics to solve MCLP with customer preference ordering. Atta, Mahapatra and Mukhopadhyay (2018) use genetic algorithm to solve MCLP and suggest that genetic algorithm approach with local refinements outperforms existing methods in terms of coverage and computational time.

Coverage problems are widely used in public facility location optimization applications. Farahani, Asgari, Heidari, Hosseininia and Goh (2012) provide a comprehensive review on coverage problems in facility location context and provide examples on how MCLP and SCLP variants are used in locating emergency medical vehicles, locating military warehouses, designing police patrol areas etc. Chow, Cheung and Yoon (2015) study on optimal quantity and locations of police stations; Karatas and Yakici (2018) provide an iterative solution approach to a multi-objective facility location problem for public emergency services. Sarıkaya, Aygüneş and Kılıç (2020) use MCLP to determine optimal locations for gendarmerie stations according to geographic and demographic characteristics of potential sites. They suggest that the minimum number of stations can be determined according to the required service level.

Enhancements in sensor technology and the usage of sophisticated sensors on road networks to collect real-time traffic information give rise to a specific type of coverage problems, Sensor Location Problem (SLP). There is extensive research on locating active (Path-ID, ALPR) and passive (loop detectors) sensors to estimate various traffic flows such as path flows, origin-destination (OD) flows, and link flows. In general, SLP formulation depends on the availability of prior traffic flow information and sensor location determination rules. Yang and Zhou (1998) introduce four different rules while determining sensor locations on a network: OD covering rule, maximal flow fraction rule, maximal flow intersecting rule, and link interdependence rule. They use these rules to develop different models for determining optimal sensor quantity and locations. While OD covering rule emphasizes the selection of locations intersecting all OD pairs at least once, maximal flow intersecting rule promotes the sensor locations with maximum vehicle flow. Wang, Ghosh and Das (2010) provide a review on SLPs and categorize problems into proximity-based localization, range-based localization and anglebased localization. Gentili and Mirchandani (2011) present an SLP framework for different sensor types (counting sensors, image sensors and license plate recognition sensors), prior information (link choice proportions, split ratios, link-route coefficients) and flows of interest (OD trips, route flows, link flows). Nine configurations are constructed with respect to sensor location determination rules, showing that certain SLP models can be relaxed to MCLP or its variants. Lessin, Lunday and Hill (2018) develop a bilevel optimization model for locating a heterogeneous set of sensors to maximize the minimum exposure of an intruder's penetration path through a defended region. Fakhouri and Soltani (2020) propose a new multi-objective model to find the optimal location of wireless sensors along highways to control traffic flow and monitor highways. Vieria, Ferrari, Ribeiro, Bahiense, Orrico Filho, Abramides and Júnior (2020) propose a progressive hybrid algorithm based on exact, heuristic and hybrid approaches embedded on a set covering framework to solve the traffic counting location problem.

This study focuses on the determination of the optimal locations of ALPR systems and contributes 
to the literature in several practical aspects: Network coverage objective is characterized as the weighted sum of two measures, vehicle coverage and road coverage. Five different weight settings are used to address the preferences of the decision-makers (DMs). Both MCLP and SLCP models are developed considering dispersion of sensors along the network. A close neighbor constraint is introduced to ensure a minimum required distance between two consecutive ALPR systems. Two real-life decisionmaking settings, CEN and DEC, are identified and the changes in the network coverages and optimal solutions under different settings are discussed. Two cities from Turkey are selected as a test problem and results are provided for both settings.

\section{The Approach}

In this study, research and publication ethics were followed. A nation-wide ALPR network that is used by law enforcement agencies is examined. The ALPR system supports law enforcement units to implement public safety practices by increasing investigative capabilities. Practices include searching for vehicles that are unregistered, stolen, involved in criminal or terrorist activities or owned by wanted people. Such an ALPR system is used for identifying, tracing, tracking, and analyzing traffic behavior at both individual and collective levels. Typically, the number of ALPR systems is limited for practical purposes. Therefore, it is assumed that there are a set of predetermined candidate locations and the problem is modelled in a discrete setting. A bi-objective integer model is developed to optimally locate finite number of ALPR systems among the candidate locations to maximize the network coverage. Then, another model is developed to find the minimum number of ALPR systems needed to achieve a specified network coverage level. Before giving the details of the approach, three significant dimensions based on which our approach is constructed are provided. First, the components of network coverage measure are characterized and the dispersion degree of ALPR locations is specified. Then, the effects of different decision-making settings are discussed. The details of each dimension are provided in Sections 3.1, 3.2 and 3.3, respectively, and the mathematical models are given in Section 3.4.

\subsection{Determinants of Network Coverage}

Due to budgetary and efficiency concerns, it would be impractical to install ALPR systems on all possible locations over the network. Generally, the aim is to achieve an efficient network, that is, the maximum network coverage (NC) with minimum number of systems. The coverage requirements are discussed with experts who are responsible for the management of ALPR network projects for law enforcement agencies in Turkey. Experts stated that the purpose of ALPR systems used by those agencies is to monitor and control traffic in the jurisdiction. To control traffic, it is important to capture maximum traffic flow for improving detection rate of minor crimes. Another important issue is tracing individual vehicles associated with major crimes. Law enforcement units utilize the gathered information (e.g. route or direction of a vehicle passing by successive/neighbor ALPR systems in a specific time period) to take action against terrorism, trafficking, auto theft, and so on. Therefore, it is important to monitor the junctions and turning points. Based on the discussions with the experts, two important measures of NC are determined as daily total number of vehicles identified and total number of different road-links monitored by the sensors in the ALPR network.

\subsection{Desired Degree of Dispersion over the Road Network}

Another important issue in determining ALPR locations is the distribution of available ALPR systems over the network. If multiple ALPR systems are located too close to each other, it results an inefficient solution in terms of distribution needs. Besides, if those ALPR systems are located on the same road-link, i.e., they are at neighbor locations both end of that road-link, then vehicle data would be duplicated at both ends. Duplicated data would result in an overestimated number of vehicles and cause misleading results in terms of NC. Therefore, a close neighbor (CN) constraint is introduced considering the distance between the locations that are on the same road-link.

\subsection{Impact of Different Decision-making Settings}

The quantity and location of ALPR systems to be installed on a network can be determined by a single central office or local authorities with respect to (w.r.t.) jurisdiction. In this study, decentralized (DEC) and centralized (CEN) cases are considered where the decision is made by local authorities and a single central authority, respectively. In the DEC case, since local authorities consider only the city- 
wide network and do not pay attention to other cities' decisions, inter-city CN cases may appear for the overall network. In the CEN case, nation-wide network is considered as a whole to achieve optimal allocation of ALPR systems to cities considering the inter-city $\mathrm{CN}$ conditions. In the study, the performances of CEN and DEC cases are compared in terms of NC.

\subsection{Mathematical Models}

Two integer linear models, MaxCover and MinInstall, are developed. The former aims to maximize $\mathrm{NC}$ for a given number of available ALPR systems whereas the latter aims to find the minimum number of ALPR systems needed to achieve a specified NC level. Although MinInstall will be discussed in Section 5.4, both models are provided in this section for the sake of completeness and readability.

Let $I$ be the index set of potential locations on the network and $P$ be the set formed by all $\mathrm{CN}$ pairs.

That is, $P=$

$\{(i, j)$ : locations $i$ and $j$ are $C N$ to eac $\square$ ot $\square$ er,$i \neq$

\section{Model MaxCover}

$\operatorname{Max} \quad w \cdot \sum_{i \in I}\left(x_{i} \cdot V C_{i}\right)+(1-w) \cdot \sum_{i \in I}\left(x_{i} \cdot R C_{i}\right)$

subject to

$\sum_{i \in I} x_{i} \leq k$

$x_{i}+x_{j} \leq 1$

$\forall(i, j) \in P$

$x_{i} \in\{0,1\}$

$\forall i \in I$

The objective function (1) maximizes the weighted sum of vehicle and road coverages of selected locations. Weighted sum method is adopted for the objective function in order to discuss the results of different weight settings between vehicle coverage and road coverage. For each location $i, V C_{i}$ is calculated as the percentage of total vehicle flow on the network. Similarly, $R C_{i}$ is calculated as the percentage of number of road-links monitored by location $i$ over total number of road-links that can be monitored by all possible locations in set $I$. (2) is the budget constraint and limits the total number of $j$ and $i, j \in I\}$ and to avoid duplication either $(i, j)$ or $(j, i)$ is included in $P$.

\section{Parameters \\ $V C_{i} \quad$ vehicle coverage of location $i \in I$ \\ $R C_{i} \quad$ road coverage of location $i \in I$ \\ $w \quad$ a parameter set by the DM, weight coefficient of $V C_{i}$ for any $i \in I$ (weight coefficient of $R C_{i}$ is $(1-w)$ ) \\ $k \quad$ total number of ALPR systems available \\ $c \quad$ targeted $\mathrm{NC}$ level}

\section{Decision Variables}

$x_{i} \quad$ binary variables that takes value of 1 if an ALPR system is located on $i, 0$ otherwise

ALPR systems available to $k$. CN constraint, (3), is used to avoid selection of close neighbor locations together in the solution. That is, when $i$ and $j$ are $\mathrm{CN}$ locations, the sum of decision variables on the lefthand side will be enforced to be less than or equal to one. This constraint checks each location pair $(i, j) \in$ $P$ and allows selecting at most one location when $i$ and $j$ are CN. Lastly, constraint (4) states that decision variables are binary. 


\section{Model MinInstall}

$\operatorname{Min} \sum_{i \in I} x_{i}$

subject to

$w \cdot \sum_{i \in I}\left(x_{i} \cdot V C_{i}\right)+(1-w) \cdot \sum_{i \in I}\left(x_{i} \cdot R C_{i}\right) \geq \mathrm{c}$

$x_{i}+x_{j} \leq 1$

$\forall(i, j) \in P$

$x_{i} \in\{0,1\} \quad \forall i \in I$

In MinInstall, only constraints (1) and (2) of MaxCover are interchanged. The objective of the MinInstall, (5), is minimizing the number of ALPR systems and it is the total number of ALPR systems available constraint (2) of the MaxCover. The minimum required $\mathrm{NC}$ constraint (6) of MinInstall is the objective of MaxCover. A new parameter $c$ is introduced in MinInstall to denote the required NC level. In (6), it can be observed that NC must be at least c. (7) is $\mathrm{CN}$ constraint and (8) is binary constraint. (7) and (8) of MinInstall are same with (3) and (4) of MaxCover, respectively.

In the rest of the paper, $\mathrm{VC}\left(\sum_{i \in \mathrm{I}} x_{i} \cdot V C_{i}\right)$ and RC $\left(\sum_{i \in I} x_{i} \cdot R C_{i}\right)$ are used to reflect the two determinants of $\mathrm{NC}$ that are daily total number of vehicles identified and number of different roadlinks monitored by the network, respectively. In addition, $w$ is introduced to reflect NC requirements for different regions. Certain areas in large networks may require specific $w$ settings due to the road network structure or traffic safety requirements. In other words, $w$ can be adjusted and set according to relative importance between VC and RC. Note that as typically done, a fixed $w$ value is used across all locations. However, if necessary, one can differentiate $w$ values for different locations simply by adding the index $i$, i.e., $w_{i}$.

$\mathrm{CN}$ constraint ensures that the solution will not contain any location pairs that are on the same roadlink with a distance less than a predetermined limit. The predetermined distance limit can be adjusted according to the size of the network and dispersion needs of possible locations on the network. CN constraint can be redefined to include maximum allowed road-distance among location pairs. If this is the case, the distance between sensor locations would have been controlled within the range of maximum and minimum. However, determining maximum allowed distance would require additional analysis on location distribution on the roadnetwork increasing the complexity of the parameter setting. For the sake of simplicity, only the minimum allowed distance is considered in this study.

\section{Case Study}

In this section, a test problem on which the performance of the approach is demonstrated and decision-making settings are presented. Numerical examples are applied to two cities in Turkey for the ALPR network used by Turkish Gendarmerie in traffic safety applications. Real-life road networks of these cities are used. First, the test problem is introduced and the reasons for the selection of the two cities are given. Then, the results of DEC and CEN cases are discussed and compared against the current state (CUR).

\subsection{Test Problem}

Turkish Gendarmerie uses a nation-wide ALPR network that consists of more than 300 ALPR installations covering more than 50 cities in Turkey. Due to Gendarmerie's area of jurisdiction, these systems are located on county and inter-city roads rather than city centres. Thanks to this ALPR network, thousands of plates are identified and examined every day to support law enforcement units in detecting and tracking vehicles associated with certain crimes.

Ankara and Kırıkkale are selected for numerical examples. Ankara, the capital city of Turkey, is the geographical hub of the road network. Kirıkkale, one of its neighbors, is a relatively small town but connecting Ankara to eastern part of Turkey. There are three main reasons for selection of these two cities:

First, the appropriate ALPR locations have already been defined for both cities by the experts. Typically, technical installation requirements, work permits, and interrelations between them make it hard to determine appropriate locations for the ALPR 
systems. Having information on available ALPR locations increases the applicability of results in real life.

Second, both cities have active ALPR systems. It provides a base for comparing the results of the experiments with the current state. This comparison will enable to make a comment on what would happen if the locations of ALPR systems were selected optimally to maximize NC.

Third and finally, Ankara and Kırkkale are neighbor cities connected by several inter-city roads (see Figure 1). For both cities, the ALPR installation locations have been determined by local authorities; providing a real-life benchmark for the DEC case.

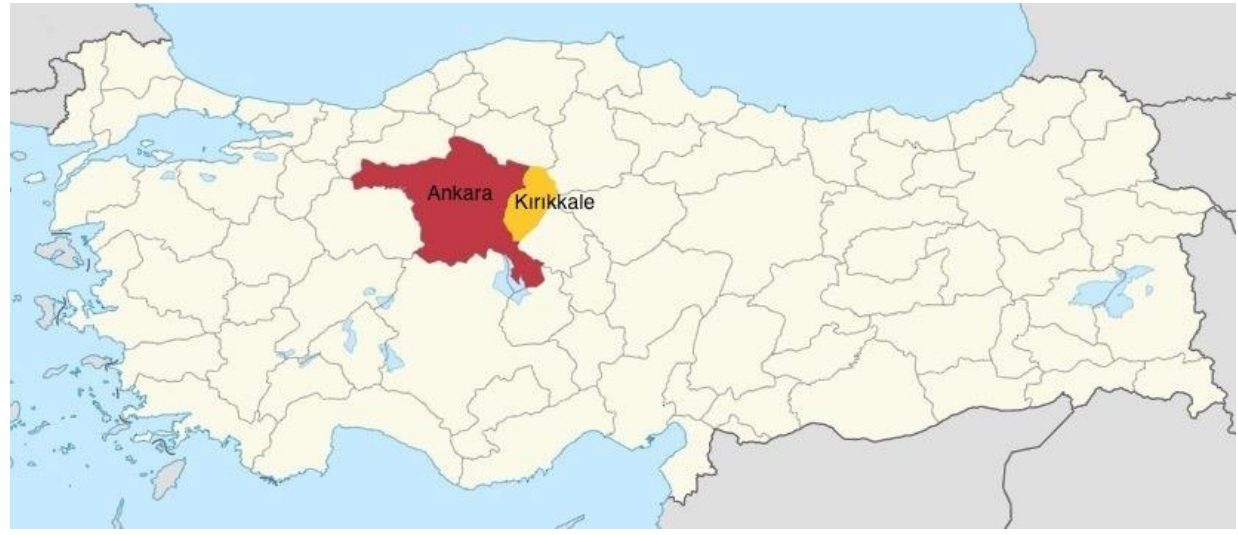

Figure 1. Locations of Ankara and Kırıkkale

\subsection{Decision-making Settings}

As stated before, in the experiments, three decisionmaking settings are considered: decentralized (DEC), centralized (CEN) and current state (CUR). For both CEN and DEC, it is assumed that there are no active ALPR systems in any city, to see the potential of the approach. In DEC, optimal ALPR locations and $\mathrm{NC}$ are examined for each city separately. Therefore, the solutions for the corresponding cities are independent. Model MaxCover is solved for all different $k$ and $w$ combinations for each city in the DEC case. Numbers of available ALPR systems for Ankara and Kırıkkale are denoted as $k^{A}$ and $k^{K}$, respectively.

In CEN, Ankara-Kırıkkale network is considered as a single road network. Therefore, a single parameter, $k^{C}$, is used as the total number of available ALPR systems for the network. Similarly, a single $w$ value is used to reflect the relative importance of VC and $\mathrm{RC}$ for the network as both cities are comparable.

In the CUR, the quantities and locations of ALPR systems to be installed in a city are determined by local authorities. Local authorities do not take the decisions of the neighboring cities into account, and this may cause inefficiency in terms of NC. In CUR, NC of the network is calculated using the locations of existing ALPR systems. Afterwards, CUR results are compared against those of the DEC and CEN settings.

In the final part, Model MinInstall is solved to find the minimum number of ALPR systems required to guarantee certain NC levels. This model is used to discuss the number of ALPR systems that are required for ensuring specified NC under two different scenarios: (i) In the presence of existing ALPR systems and (ii) Over an empty network. An analysis is conducted on the minimum number of ALPR installations needed for improving existing NC.

\section{Results and Discussions}

In this section, the results of numerical examples for DEC Ankara and Kırıkkale cases, CEN case and CUR analysis will be provided. Then, the improvement of existing NC will be discussed. For all cases, VC and RC values are calculated using Traffic Volume Maps (2016) issued by General Directorate of Highways. These maps provide the link-flows obtained by vehicle counting sensors located throughout the road network. The calculation procedure for $\mathrm{VC}$ and RC parameters is provided in Appendix A. Five different $w(0.1,0.3,0.5,0.7,0.9)$ values are used to represent coverage preference of the DM. Note that different $w$ settings reflect different scenarios in terms of the trade-off between $\mathrm{VC}$ and $\mathrm{RC}$, i.e., 
different preference structures for the DM. The models are implemented in GAMS ${ }^{\circledR}$ v23.9. CPLEX is used as the solver and the absolute (optimality) gap is set to zero. Please see the model details and solver performance in the following table.

Table 1

Model details and solver performance

\begin{tabular}{lccc} 
& DEC - Ankara & DEC - Kirkkkale & CEN \\
\hline Number of DVs & 13 & 6 & 19 \\
Number of constraints & 6 & 3 & 9 \\
CPU (seconds)* & 0.0409 & 0.0391 & 0.0436 \\
\hline
\end{tabular}

* Average duration

\subsection{Decentralized Case (DEC)}

For Ankara, a total of 176,132 vehicles in a day and 37 different road-links can be monitored by 13 available ALPR locations; whereas a total of 72,058 vehicles in a day and 20 different road-links can be monitored by six available ALPR locations in Kırıkkale.

Recall that $w$ is used to differentiate the importance of VC and RC. If VC and RC have positive correlation, then a location with high VC is expected to have high

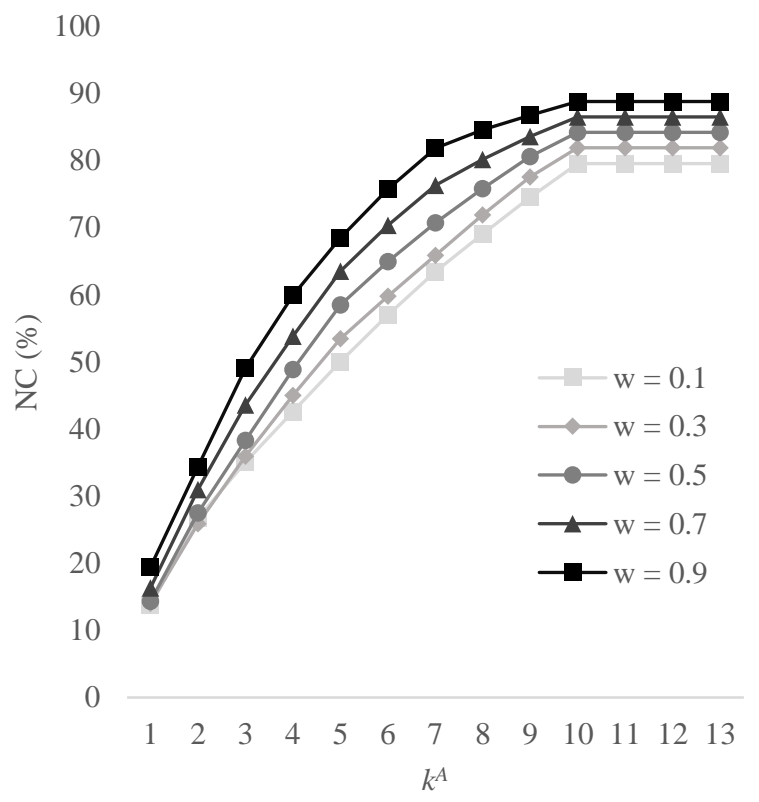

(a) DEC Ankara
$\mathrm{RC}$ as well. In this case, $w$ cannot depict relative importance of the two parameters and optimal solutions may not change at all even $w$ changes dramatically. The correlation coefficients between VC and RC are calculated as 0.06 and -0.11 for Ankara and Kırıkkale, respectively indicating that there is no strong correlation between these parameters for either city. This also indicates that VC and RC are not conflicting measures.

The DEC Ankara and Kırıkkale problems are solved for 13 different $k^{A}$ and six different $k^{K}$ values, respectively. NC values for different parameters are illustrated in Figure 2.

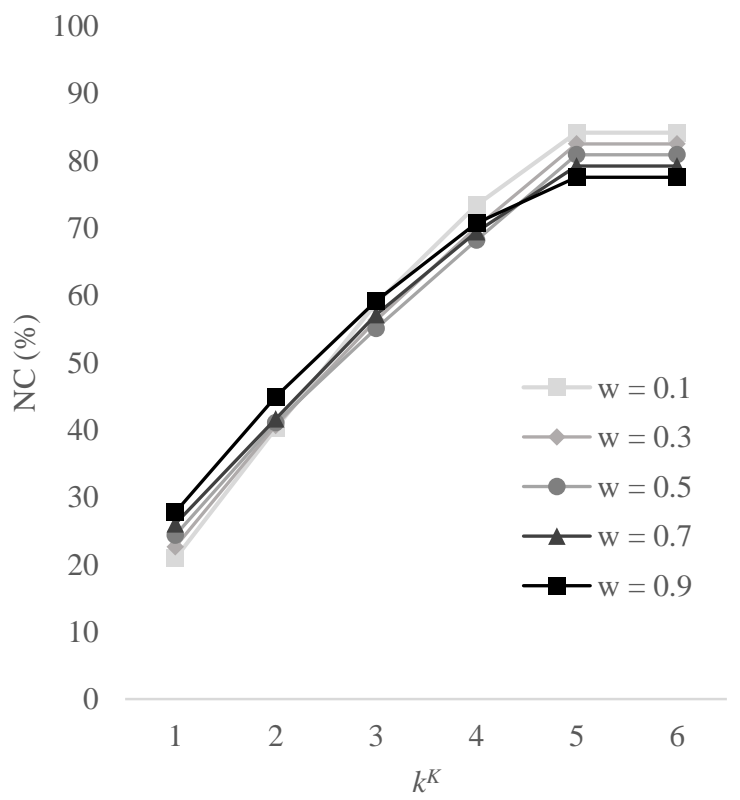

(b) DEC Kırıkkale

Figure 2. Demonstration of Optimal NC for the DEC Case for Different $\boldsymbol{w}$ Values 
Due to CN constraint, at most 10 out of 13 ALPR locations can be utilized for Ankara. Similarly, at most five out of six locations can be selected for Kirlkkale. The maximum NC values are between $79.5 \%$ and $88.8 \%$ for Ankara and $77.6 \%$ and $84.2 \%$ for Kirıkkale for different $w$ values.

It can be inferred from Figure 2 that as $w$ increases NC increases for any $k^{A}$. The range of VC is 0.20 (0.01-0.21) while range of RC is 0.09 (0.05-0.14) for the locations in Ankara. As $w$ increases, locations with higher VC are utilized and NC values with higher $w$ dominates those with lower $w$. On the other hand, this trend cannot be observed in Kirıkkale case. In Kirlkkale, for $k^{K} \leq 2$, NC is larger when $w=0.9$; however, for $k^{K} \geq 4, \mathrm{NC}$ is the highest for $w=0.1$. The reason is that the impact of $w$ for different $k$ values depends on VC and RC. That is, for some VC and RC combinations, higher $w$ may generate higher NC regardless of $k$ as in the case of Ankara whereas for some combinations, it may turn out to be the opposite.

\subsection{Centralized Case (CEN)}

In this setting, Ankara and Kirıkkale will be considered as a single network. In CEN, the parameters of Ankara and Kırıkkale $\left(k^{A}\right.$ and $\left.k^{K}\right)$ are combined and totally 19 ALPR locations are available. With these 19 locations 248,190 vehicles in a day and 57 different road-links can be monitored. It should be noted that VC and RC calculations are based on city totals in the DEC case. These values are recalculated w.r.t. network totals in the CEN case. Since the network totals are larger than city totals, all VC and RC parameters in CEN are smaller than those in DEC. While comparing with DEC, CEN parameters will be used to make a fair comparison.

There are two main differences between DEC and CEN settings. First, in DEC, the quantity and location of ALPR systems are determined independently. In CEN, on the other hand, total number of ALPR systems available, $k^{C}$, can be allocated optimally considering the overall network. Second, in DEC, local DMs eliminate utilization of inner-city $\mathrm{CN}$ locations considering only their jurisdiction. However, in CEN, both inner-city and inter-city CN locations are addressed.

CEN problem is solved for 19 different $k^{C}$ (from 1 to 19) and optimal NC values for different $k^{C}$ are demonstrated in Figure 3.

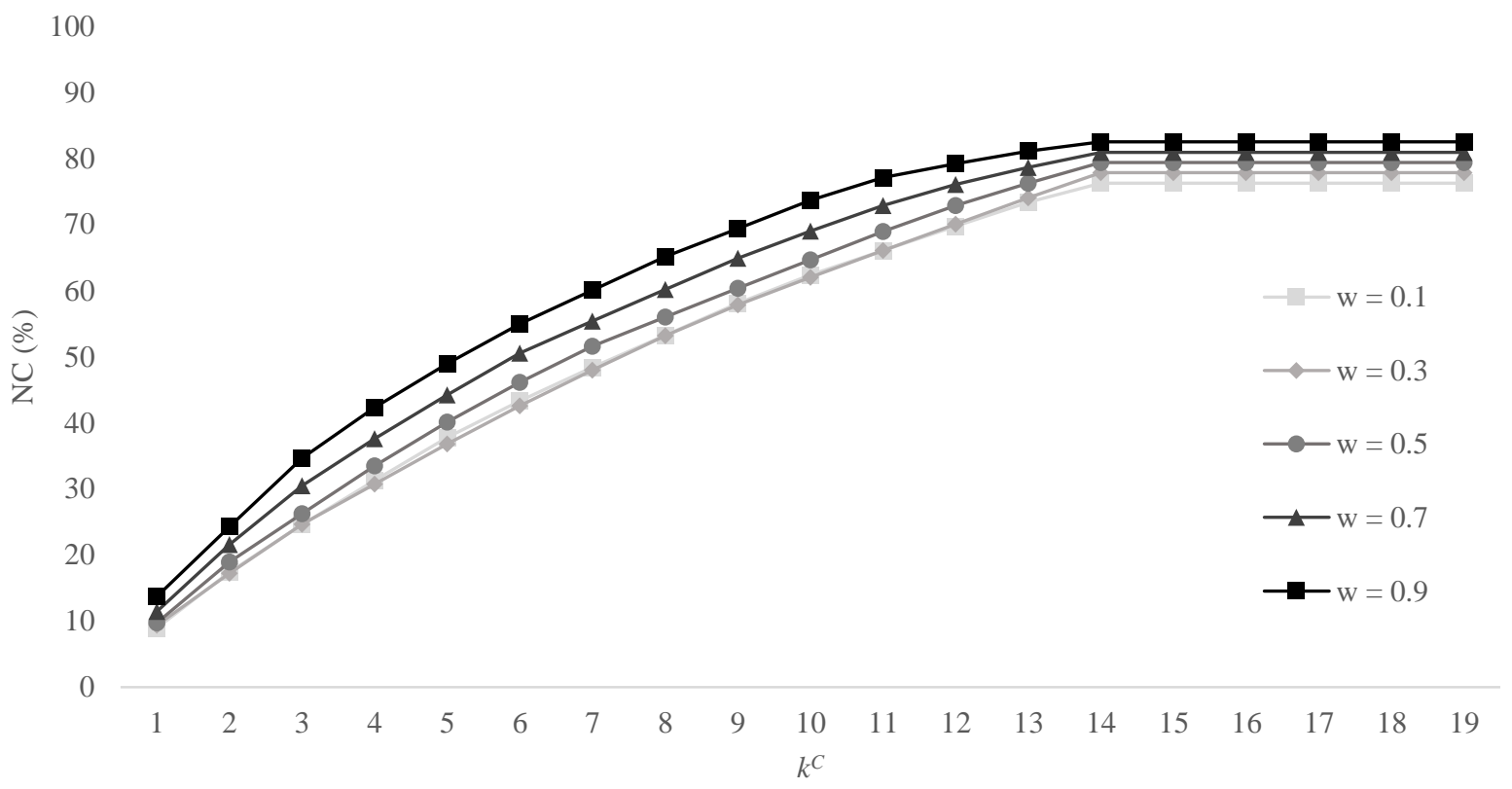

Figure 3. Demonstration of Optimal NC for the CEN Case for Different $w$ Values 
It can be observed from Figure 3 that the optimal NC of CEN is between $76.2 \%$ and $82.5 \%$. In CEN, at most 14 locations can be utilized while it is totally 15 in DEC. It is due to an inter-city CN location pair between Ankara and Kırıkkale. In the DEC case the inter-city $\mathrm{CN}$ locations can be selected as the cities are treated separately; however, in the CEN case, these locations cannot be selected simultaneously. As in the DEC Ankara case, the NC values generated with higher $w$ dominate those with lower $w$ for any $k^{C}$. The range of $\mathrm{VC}$ is $0.14(0.01-0.15)$ while range of $\mathrm{RC}$ is 0.05 (0.04-0.09) for the CEN case. Thus, when $w$ increases, locations with high VC are utilized and the corresponding optimal solution results in higher $\mathrm{NC}$ while the NC loss due to RC change is smaller.

While comparing DEC and CEN, if inter-city $\mathrm{CN}$ is utilized in DEC, then VC penalty is applied. It is assumed that VC is duplicated in CN locations and only the maximum $\mathrm{VC}$ of the $\mathrm{CN}$ locations is taken into calculations. RC is a parameter concerned with determining directions of vehicles rather than the amount of flow. Therefore, RC of CN locations is calculated as it is for any other location pair.
To make an overall DEC and CEN comparison, all possible $k^{A}$ and $k^{K}$ configurations that compose $k^{C}$ ranging from 2 to 14 are examined and results are compared under different $w$ values. For each $w, 62$ different $k^{A}$ and $k^{K}$ DEC configurations are compared to the corresponding CEN case. The results show that CEN solutions outperform the DEC ones. For 282 out of 310 cases, CEN setting results better NC than DEC. For 21 cases, CEN and DEC yield the same NC. Only in seven cases, DEC performs better than CEN (see Appendix B for these cases). Such cases are only observed for $k^{C} \geq 11$ and $w=0.1$ where importance of RC is the highest. For these specific configurations, CEN solution includes lowNC locations (cannot select intercity $\mathrm{CN}$ locations which would have higher NC due to road coverage) since there is a limited set of locations available for $k^{C} \geq 11$. On the other hand, DEC solution leverages higher-NC locations which are intercity CNs.

When all possible combinations of $k^{A}$ and $k^{K}$ are compared with the corresponding $k^{C}$ (a total of 62 configurations), an overall difference between CEN and DEC optimal solutions are summarized in Figure 4.

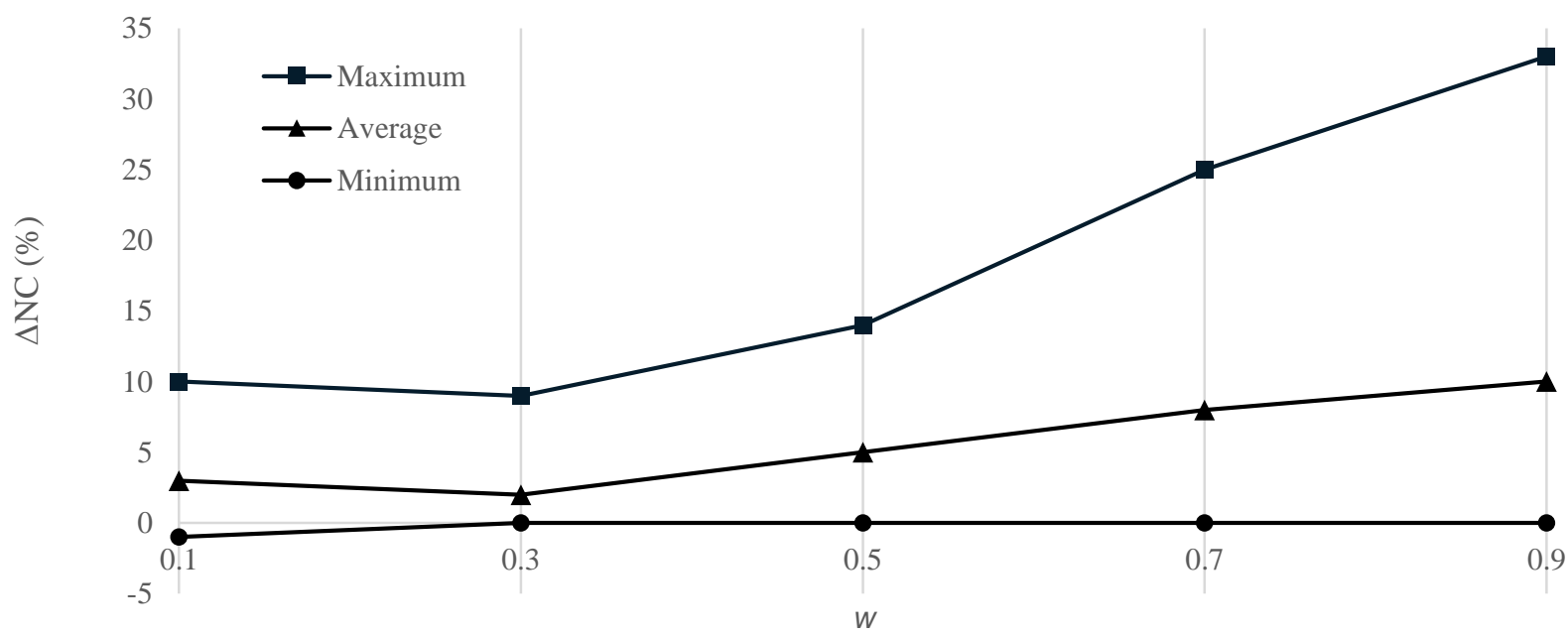

Figure 4. Impact of $\boldsymbol{w}$ on NC Differences between CEN and DEC Cases
In Figure 4, y-axis, $\triangle \mathrm{NC}$, shows the difference between NC values generated by CEN and DEC optimal solutions, and the maximum, minimum and average of $\triangle \mathrm{NC}$ values are provided. As it can be seen from Figure 4, for almost all cases CEN solution has better NC than DEC solution. There are two main reasons: First, in the DEC setting, cities may not find the best configuration for $k^{A}$ and $k^{K}$ without coordination. Second, DEC solution ignores the existence of inter-city $\mathrm{CN}$. Notice that as the 156 importance of VC increases, the difference becomes more apparent.

The average difference increases from $2.6 \%$ to $9.9 \%$ while $w$ increases from 0.1 to 0.9 . For $w=0.1$, the number of road-links monitored is more important than the number of vehicles detected in a day. When compared to DEC, CEN optimal locations can detect 2,417 fewer vehicles but can monitor 1.7 more roadlinks on average. On the other hand, when $w=0.9$, 
27,544 more vehicles in a day can be detected without giving up any RC.

\subsection{Current State (CUR)}

In this part, CUR of the Ankara-Kırıkkale network is analyzed and compared to CEN and DEC optimal solutions with same parameters. In each city there are three active ALPR systems. Experts state that for Ankara-Kırıkkale region, the number of vehicles detected in a day is more important than number of road-links monitored. Therefore, the analysis is conducted for $w=0.7$ and $w=0.9$. CUR and corresponding DEC $\left(k^{A}=k^{K}=3\right)$ and CEN $\left(k^{C}=6\right)$ solutions are compared in terms of NC in Table 2.

Table 2

NC Comparison for CUR, DEC and CEN Cases

\begin{tabular}{|c|c|c|c|c|c|c|}
\hline Setting & $\Sigma \mathrm{V}$ & $\Sigma \mathrm{R}$ & $\begin{array}{l}\Sigma \mathrm{VC} \\
(\%) \\
\end{array}$ & $\Sigma \mathrm{RC}(\%)$ & $\begin{array}{c}\mathrm{NC}(\%) \\
w=0.7\end{array}$ & $\begin{array}{c}\text { NC }(\%) \\
w=0.9\end{array}$ \\
\hline CUR & 102,685 & 20 & 41.4 & 35.1 & 39.5 & 40.7 \\
\hline DEC & 113,853 & 19 & 45.9 & 33.3 & 42.1 & 44.6 \\
\hline DEC - CUR & 11,168 & -1 & 4.5 & -1.8 & 2.6 & 3.9 \\
\hline CEN & 141,764 & 20 & 57.1 & 35.1 & 50.5 & 54.9 \\
\hline CEN - CUR & 39,079 & 0 & 15.7 & 0.0 & 11.0 & 14.2 \\
\hline CEN - DEC & 27,911 & 1 & 11.2 & 1.8 & 8.4 & 10.3 \\
\hline
\end{tabular}

In Table $2, \Sigma \mathrm{V}$ and $\Sigma \mathrm{R}$ represent total number of vehicles and total number of road-links that can be covered by the corresponding optimal solution, respectively. Optimal solutions for both CEN and DEC do not change for $w=0.7$ and $w=0.9$ cases. However, NC values change w.r.t. $w$. The DEC optimal solution would have improved network coverage by 2.6-3.9\% only by selecting the optimal locations for given $k^{A}$ and $k^{K}$. By CEN optimal solution, NC of CUR would have been improved by $11.0-14.2 \%$. Also notice that, the CEN optimal solution generates 8.4$10.3 \%$ higher NC than that of the DEC optimal solution. There are two major sources for this improvement: (i) optimal allocation of ALPR systems over the network and (ii) addressing inter-city CN locations.

In Table 2, it can be observed that the optimal solution of CEN has an absolute advantage over those of DEC and CUR. When compared to CUR, the optimal solution of DEC has better VC and worse RC values; however, for both $w$ values NC values for DEC are better than those of CUR. The reason is that for larger $w$ values where VC is more important, the optimal solution of DEC gives up RC for higher VC to maximize NC. If $w$ is set to less than 0.28 , which is the breakeven point between NC values of CUR and DEC optimal solution, then CUR would yield better NC values than the DEC optimal solution. In the CEN case both VC and RC values are at least as high as those of CUR; thus, NC of CEN is always greater than those of CUR for all $w$ values. After installation, it is very costly to relocate an ALPR system. Therefore, the optimal locations suggested by the CEN case should have been utilized for maximizing $\mathrm{NC}$ in the long run. $\mathrm{NC}$ comparison for different settings is illustrated in Figure 5. 


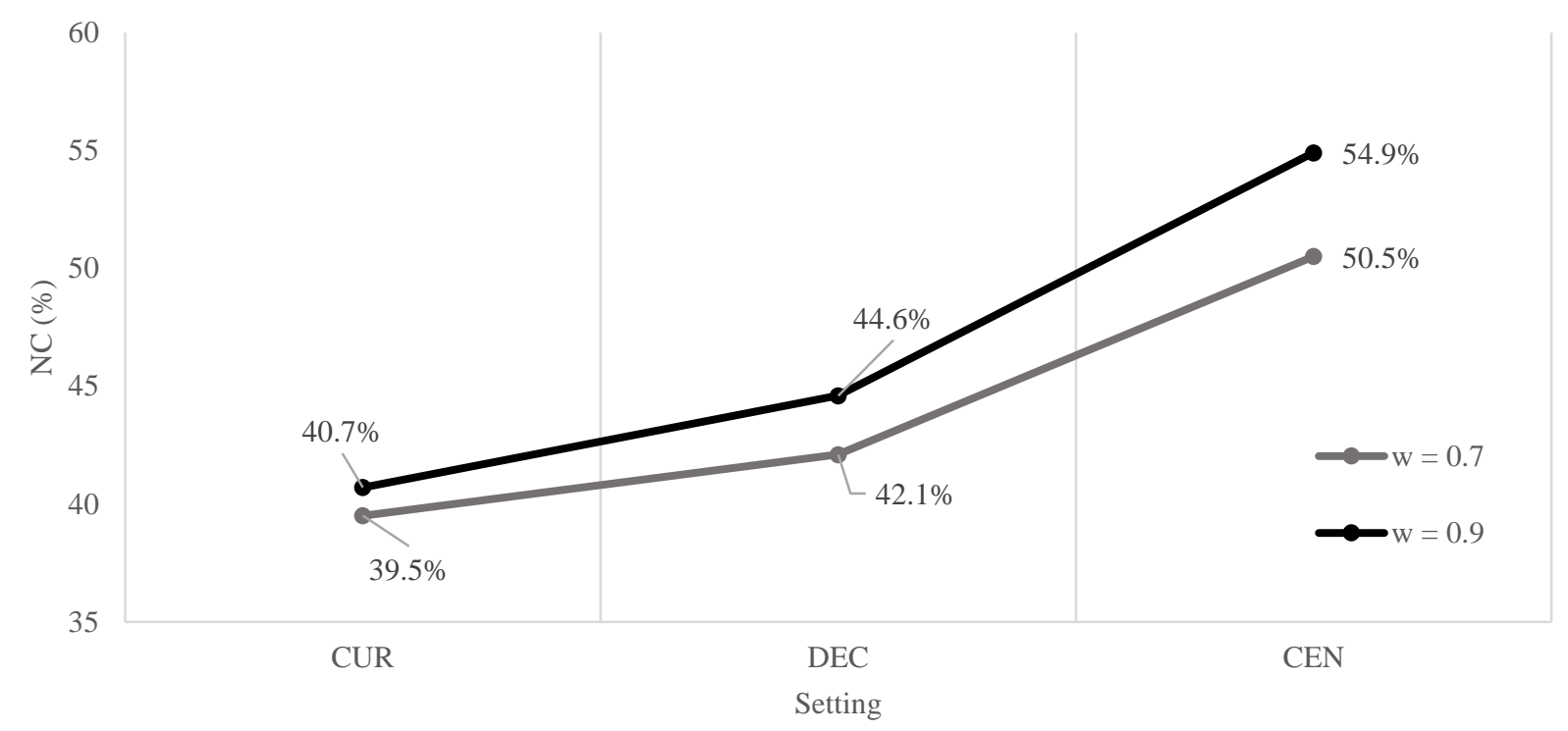

Figure 5. NC Comparison w.r.t. Solution Settings, for $k^{A}=k^{K}=3$ and $k^{C}=6$

\subsection{Improvement of Existing NC}

Up to this part, CEN and DEC problems are solved assuming that there are no ALPR systems installed on the network, for the sake of simplicity. However, relocating or shutting down an ALPR system that has already been installed is very costly. Therefore, existing systems should be considered while deciding on the quantity and location of ALPR systems. NC of existing network is $39.5 \%$ where $w=0.7$ and $k=6$. How many more ALPR systems are needed to achieve $60 \%$ or $70 \%$ NC for the same $w$ and $k$ setting? Where to locate these additional ALPR systems? Assuming that there were no current ALPR systems on the network, would it change the total number of installations for achieving specified coverage levels? To answer these questions,
MinInstall which is an SCLP variant of MaxCover is solved.

MinInstall aims to find the minimum number of ALPR systems needed to achieve a specified NC level, $c$. The problem is solved for different $c$ and $w$ values in the CEN setting. First, it is assumed that the network does not have pre-existing ALPR systems installed. Then, the active ALPR systems located in Ankara and Kırıkkale are taken into consideration and the problem is solved again. In the second case, additional location constraints are used in the model to define pre-existing ALPR systems.

The two set of solutions obtained are used to compare the impact of active ALPR systems located on the network. In other words, the difference between building from empty network and cost of upgrading the existing sub-optimal network are examined. The results are given in Table 3. 
Table 3

Minimum Number of ALPR Systems Needed to Ensure Coverage Level $c$

\begin{tabular}{|c|c|c|c|c|c|c|c|c|c|c|}
\hline \multirow[b]{2}{*}{$w$} & \multirow[b]{2}{*}{ Scenario } & \multicolumn{9}{|c|}{$c(\%)$} \\
\hline & & 40 & 45 & 50 & 55 & 60 & 65 & 70 & 75 & 80 \\
\hline \multirow{2}{*}{0.1} & Empty network & 6 & 7 & 8 & 9 & 10 & 11 & 13 & 14 & - \\
\hline & Upgrade & 7 & 8 & 8 & 9 & 10 & 11 & 13 & 14 & - \\
\hline \multirow{2}{*}{0.3} & Empty network & 6 & 7 & 8 & 9 & 10 & 11 & 12 & 14 & - \\
\hline & Upgrade & 7 & 8 & 8 & 9 & 10 & 12 & 13 & 14 & - \\
\hline \multirow{2}{*}{0.5} & Empty network & 5 & 6 & 7 & 8 & 9 & 11 & 12 & 13 & - \\
\hline & Upgrade & 7 & 7 & 8 & 9 & 11 & 12 & 13 & - & - \\
\hline \multirow{2}{*}{0.7} & Empty network & 5 & 6 & 6 & 7 & 8 & 10 & 11 & 12 & 14 \\
\hline & Upgrade & 7 & 7 & 8 & 9 & 10 & 12 & 13 & - & - \\
\hline \multirow{2}{*}{0.9} & Empty network & 4 & 5 & 6 & 7 & 7 & 8 & 10 & 11 & 13 \\
\hline & Upgrade & 6 & 7 & 8 & 9 & 10 & 11 & 14 & - & - \\
\hline
\end{tabular}

Recall that for Ankara-Kırıkkale network six ALPR systems have been already installed. NC values of CUR are $39.5 \%$ and $40.8 \%$ for $w=0.7$ and $w=0.9$, respectively. To upgrade existing $\mathrm{NC}$ to $50 \%$, two more ALPR systems must be utilized making a total of eight ALPR systems for each $w$ value. If the network was empty, it would be possible to achieve $50 \%$ NC with six ALPR systems.

When RC is more important than $\mathrm{VC}$ for a DM (i.e., $w=0.1$ or $w=0.3$ ), the minimum number of ALPR systems required for a specific $c$ value does not change dramatically whether the network is built from empty or upgraded. For the cases where VC is at least as important as RC $(w \geq 0.5)$ the difference increases. Even though both scenarios are solved in the CEN setting, it is expected that built from an empty network would yield better NC than upgrading the existing ALPR systems of CUR of which does not take inner and inter-city $\mathrm{CN}$ constraint into consideration. However, the increase in minimum number of ALPR systems required is directly associated with cost increase and for extreme cases, the cost increase reaches up to $50 \%$. The results indicate that to build a cost effective ALPR network, CEN setting should be used from at the beginning. In other words, even though the best method is used, pre-existing sub-optimal locations would cause substantial upgrade costs.

\section{Conclusions}

In this study, a methodological approach is provided to locate ALPR systems optimally in a road network under different decision-making settings, CEN and
DEC. The study contributes to the literature in several practical aspects. Traffic volume maps issued by the General Directorate of Highways are used to obtain number of vehicles and road-links and suggest a calculation method for $\mathrm{VC}$ and $\mathrm{RC}$ parameters. A weight coefficient, $w$, is introduced to distinguish the impact of VC and RC for different cities. Finally, a CN constraint that has an effect on distribution and dispersion of ALPR sensors over the network is introduced. Solutions generated for DEC, CEN and CUR settings are compared and an analysis on improvement of existing NC is provided.

The CEN optimal solution is expected to generate higher NC than DEC optimal solution since in the CEN setting inter-city $\mathrm{CN}$ locations and the best allocation of ALPR systems over cities are taken into account. NC values of the current state, CUR, for both cities are lower compared to DEC and CEN solutions. When pre-existing ALPR systems are considered, it turns out that upgrading an existing sub-optimal network requires higher number of systems than building from an empty network for the same coverage level.

Two neighbor cities from Turkey are selected and examined in the pilot study. However, the proposed methodology can be extended for any road network and can be used for other cities and countries. However, it should be noted that when the problem size increases, the computational time may be high or even finding a solution may be impossible due to the NP-hard property of the problem. In such cases, heuristic methods can be applied to achieve a solution in reasonable time. Ankara and Kirıkkale road networks represent average cases and hence, 
the method can be implemented directly for most of the cities in Turkey. For the cities requiring special treatment, on the other hand, to address such requirements some adjustments/modifications may be necessary before the implementation of the method. For example, Istanbul is one of the most crowded cities in the world and the terrestrial trade hub of Turkey. Several adjustments should be made considering the high-volume trade routes and new parameters may be introduced such as a crowdedness measure. Another example can be the case of Diyarbakır. Diyarbakır is one of the biggest cities in Turkey that is located south east of the country and law enforcement units are struggling with trafficking, terrorism, and human smuggling. For such a road network, additional parameters concerning these mass crimes should be incorporated for available ALPR locations.

This study suggests an approach for selecting optimal ALPR locations for a sample network but has some assumptions and limitations to be addressed in further studies. Here stationary daily average traffic volume and road network are assumed. Potential changes in road network and drivers' route preferences would affect optimal ALPR locations. The $\operatorname{DM}(\mathrm{s})$ of this problem may not have the authority to design a road network. However, drivers' route selection can be analyzed through a trend analysis over the concerned time period to forecast future traffic volumes of road-links. Long term averages of vehicle volumes can be used in VC calculation.

The ALPR systems examined in this study have fixed locations. There are mobile ALPR (MALPR) systems that can be mounted on vehicles or even on bikes, and can be located on any road at any time. However, since their mobility would require dynamic location determination, MALPR systems are not included in this study. In further studies, mobile and fixed ALPR systems may be combined to determine fixed locations for traditional ALPR and dynamic locations for MALPR through NC maximization in a progressive manner.

As a final remark, reorganization of this study considering human crowdedness rather than traffic volume and number of road-links is a subject of future research. ALPR locations can be set according to closeness to densely populated areas and public places as hospitals, schools and industrial regions.

\section{Contributions of Researchers}

In this study, the contributions of Buğra GÖR are conceptualization, methodology, software, interpretation of the results, investigation, writing, visualization, and the contributions of Gülşah KARAKAYA are conceptualization, software, interpretation of the results, writing.

\section{Conflict of Interest}

The authors declare no conflict of interest.

\section{References}

Atta, S., Mahapatra, P. R. S. \& Mukhopadhyay, A. (2018). Solving maximal covering location problem using genetic algorithm with local refinement. Soft Computing, 22(12), 3891-3906. Doi: $\quad$ https://doi.org/10.1007/s00500-0172598-3

Castillo, E., Menéndez, J. M. \& Jiménez, P. (2008). Trip matrix and path flow reconstruction and estimation based on plate scanning and link observations. Transportation Research Part B: Methodological, 42(5), 455-481. Doi: https://doi.org/10.1016/j.trb.2007.09.004

Coaffee, J. (2004). Rings of steel, rings of concrete and rings of confidence: designing out terrorism in central London pre and post September 11th. International Journal of Urban and Regional Research, 28(1), 201-211. Doi: https://doi.org/ 10.1111/j.0309-1317.2004.00511.x

Chow, A. H., Cheung, C. Y. \& Yoon, H. T. (2015). Optimization of police facility locationing. Transportation Research Record, 2528(1), 60-68. Doi: https://doi.org/ $\underline{10.3141 / 2528-07}$

Díaz, J. A., Luna, D. E., Camacho-Vallejo, J. F. \& CasasRamírez, M. S. (2017). GRASP and hybrid GRASPTabu heuristics to solve a maximal covering location problem with customer preference ordering. Expert Systems with Applications, 82, 67-76. Doi: https://doi.org/10.1016/ j.eswa.2017.04.002

Fakhouri, A. A. \& Soltani, R. (2020). Multi-objective robust optimization for the traffic sensors location problem. IEEE Access. Doi: http://dx.doi.org/10.1109/ACCESS.2020.3047045 
Farahani, R. Z., Asgari, N., Heidari, N., Hosseininia, M. \& Goh, M. (2012). Covering problems in facility location: A review. Computers \& Industrial Engineering, 62(1), 368-407. Doi: https://doi.org/10.1016/i.cie.2011.08.020

General Directorate of Highways. Traffic Volume Map (2016) Retrieved from: http://www.kgm.gov.tr/SiteCollectionDocument s/KGMdocuments/Trafik/trafikhacimharitasi/2 016HacimHaritalari/Hacim2016Devlet.pdf. Accessed 31 August 2018.

Gentili, M. \& Mirchandani, P. (2011). Survey of models to locate sensors to estimate traffic flows. Transportation Research Record: Journal of the Transportation Research Board, (2243), 108116. Doi: https://doi.org/10.3141/2243-13

Jia, H., Ordonez, F. \& Dessouky, M. M. (2007). Solution approaches for facility location of medical supplies for large-scale emergencies. Computers \& Industrial Engineering, 52(2), 257-276. Doi: https://doi.org/10.1016/j.cie.2006.12.007

Karatas, M. \& Yakıcı, E. (2018). An iterative solution approach to a multi-objective facility location problem. Applied Soft Computing, 62, 272-287. Doi: https://doi.org/10.1016/i.asoc.2017.10.035

Lessin, A. M., Lunday, B. J. \& Hill, R. R. (2018). A bilevel exposure-oriented sensor location problem for border security. Computers \& Operations Research, 98, 56-68. Doi: https://doi.org/10.1016/j.cor.2018.05.017

Sarıkaya, H. A., Aygüneş, H. \& Kılıç, A. (2020). Determining the locations of the gendarmerie stations using the maximal covering method. Journal of Industrial Engineering, 31(1), 28-47.

Sousanis, J. (2011). World vehicle population tops 1 billion units. Wards Auto, 15. Retrieved from: https://www.wardsauto.com/newsanalysis/world-vehicle-population-tops-1billion-units.
United States Department of Justice. Office of Justice Programs. Bureau of Justice Statistics. Law Enforcement Management and Administrative Statistics (LEMAS), 2007. ICPSR31161-v1. Ann Arbor, MI: Inter-university Consortium for Political and Social Research [distributor], 201107-07. Doi: https://doi.org/10.3886/ ICPSR31161.v1

United States Department of Justice. Office of Justice Programs. Bureau of Justice Statistics. Law Enforcement Management and Administrative Statistics (LEMAS), 2013. ICPSR36164-v2. Ann Arbor, MI: Inter-university Consortium for Political and Social Research [distributor], 201509-22. Doi: https://doi.org/10.3886/ ICPSR36164.v2

Vieira, B. S., Ferrari, T., Ribeiro, G. M., Bahiense, L., Orrico Filho, R. D., Abramides, C. A. \& Júnior, N. F. R. C. (2020). A progressive hybrid set covering based algorithm for the traffic counting location problem. Expert Systems with Applications, 160, 113641. Doi: https://doi.org/10.1016/i.eswa. $\underline{2020.113641}$

Voelcker, J. (2014). 1.2 Billion Vehicles On World's Roads Now, 2 Billion By 2035: Report. Green Car Reports, July,29. Retrieved from: https://www.greencarreports.com/news/1093 560 1-2-billion-vehicles-on-worlds-roads-now2-billion-by-2035-report

Wang, J., Ghosh, R. K. \& Das, S. K. (2010). A survey on sensor localization. Journal of Control Theory and Applications, 8(1), 2-11. Doi: https://doi.org/10.1007/s11768-010-9187-7

Yang, H. \& Zhou, J. (1998). Optimal traffic counting locations for origin-destination matrix estimation. Transportation Research Part B: Methodological, 32(2), 109-126. Doi: https://doi.org/10.1016/S01912615(97)00016-7 


\section{VC and RC Calculation Procedure}

1. Traffic volume maps for provincial roads and state roads are combined for each location.
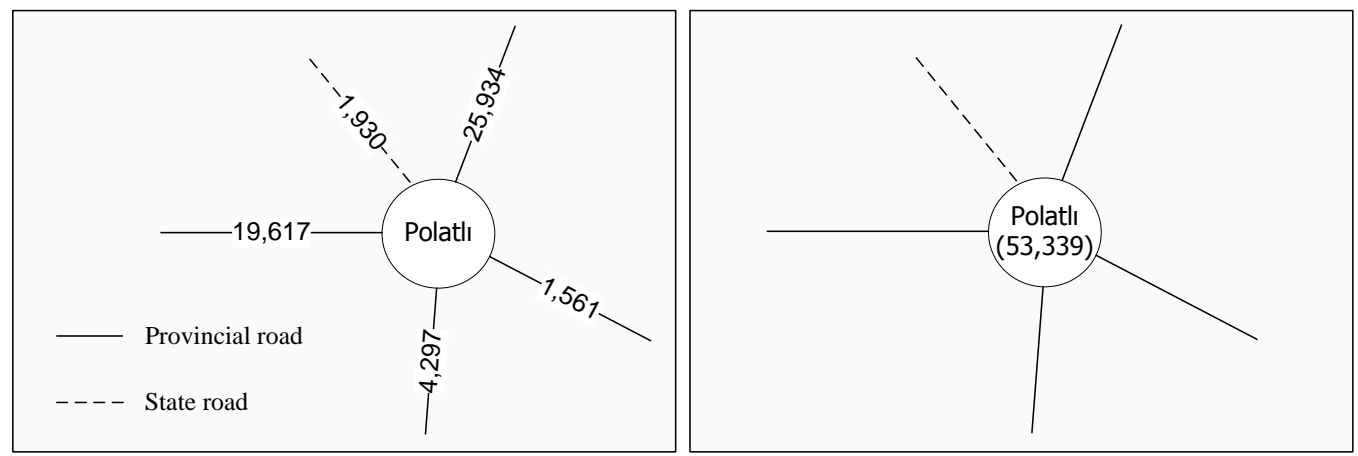

Figure 6. Connected Road-link with Flow Volumes (Left) and Total Vehicle Count (Right) for Polatlı

2. The number of road-links connected to Polatlı is counted. For Polatll, the number of road-links monitored, Rolatl, is five as it is seen in Figure 6.

3. The numbers of vehicles detected by nearest counting stations for all road-links connected to the location are added up. For Polatl, total number of vehicles is 53,339 as illustrated in Figure 6.

4. The total vehicle count is divided by 2 . The vehicle counts observed on links are bidirectional. It is assumed that half of the vehicles detected on links are directed to Polatl while the rest is directed the opposite direction. As a result, the daily number of vehicles passing by Polatlı, $\mathrm{V}_{\text {Polatll, }}$ is calculated as 26,670 .

5. For all possible ALPR locations on the network, first 4 steps are followed and total number of vehicles $\left(\sum_{i} V_{i}\right)$ and total number of road-links $\left(\sum_{i} R_{i}\right)$ that can be covered by all possible ALPR locations are found for the network.

6. $V C_{i}$ and $R C_{i}$ for any location $i$ is calculated according to the formulas given below:

$$
V C_{i}=V_{i} / \sum_{i} V_{i} \quad R C_{i}=R_{i} / \sum_{i} R_{i}
$$

\section{A. Cases DEC NC is Higher than CEN NC}

Table 4

Cases where DEC Solutions Generate Higher NC than CEN Solutions, $w=0.1$

\begin{tabular}{cc|ccc|c|ccc|cc|c}
\hline & & \multicolumn{3}{|c|}{ DEC } & & \multicolumn{3}{c|}{ CEN } & & \\
\hline$k^{A}$ & $k^{K}$ & $\Sigma V C(\%)$ & $\Sigma \mathrm{RC}(\%)$ & $\mathrm{NC}(\%)$ & \multirow{2}{*}{$k^{C}$} & $\begin{array}{c}\Sigma \mathrm{VC} \\
(\%)\end{array}$ & $\begin{array}{c}\Sigma \mathrm{RC} \\
(\%)\end{array}$ & $\begin{array}{c}\mathrm{NC} \\
(\%)\end{array}$ & $\begin{array}{c}\Delta \mathrm{VC} \\
(\%)\end{array}$ & $\begin{array}{c}\Delta \mathrm{RC} \\
(\%)\end{array}$ & $\begin{array}{c}\Delta \mathrm{NC} \\
(\%)\end{array}$ \\
\hline 7 & 4 & 61.6 & 66.7 & 66.2 & 11 & 60.3 & 66.7 & 66.0 & -1.2 & 0.0 & -0.1 \\
8 & 4 & 66.9 & 70.2 & 69.8 & 12 & 65.6 & 70.2 & 69.7 & -1.3 & 0.0 & -0.1 \\
7 & 5 & 66.8 & 70.2 & 69.8 & 12 & 65.6 & 70.2 & 69.7 & -1.2 & 0.0 & -0.1 \\
9 & 4 & 71.3 & 73.7 & 73.4 & 13 & 69.9 & 73.7 & 73.3 & -1.3 & 0.0 & -0.1 \\
8 & 5 & 72.1 & 73.7 & 73.5 & 13 & 69.9 & 73.7 & 73.3 & -2.2 & 0.0 & -0.2 \\
10 & 4 & 71.3 & 77.2 & 76.6 & 14 & 83.2 & 75.4 & 76.2 & 12.0 & -1.8 & -0.4 \\
9 & 5 & 76.5 & 77.2 & 77.1 & 14 & 83.2 & 75.4 & 76.2 & 6.8 & -1.8 & -0.9
\end{tabular}


In Table 4, all DEC solutions include CN locations. Recall that, when $\mathrm{CN}$ locations are implemented in neighboring cities, maximum VC of those locations is considered while calculating NC. On RC calculation, nothing is omitted, and road-links covered by the $\mathrm{CN}$ locations are added up.

Since there are very few alternative locations to switch for $k^{c} \geq 11$, CEN solution had to give up a location that has a higher weighted score than remaining alternatives due to $\mathrm{CN}$ constraint. For the first five cases, the negative impact of VC lost in DEC solution is smaller than the negative impact of switching to a worse-scored location in CEN solution.
Specifically, while DEC solution uses locations 9 (Ankara) and 14 (Kırıkkale) that are CN, CEN solution had to switch location 9 to a location with lower VC. In the last two cases, DEC solutions incorporating inter-city $\mathrm{CN}$ have smaller VC values than those of the CEN solutions. However, those CN locations have high RC that compensates the lost in VC. Considering $w=0.1$, the impact of lower $\mathrm{RC}$ results in lower NC in CEN case.

The cases that DEC NC is better than the CEN NC are very rare and only observed for high $k^{C}$ and low $w$ values. 